\title{
Intorno al brutto. Una categoria controversa
}

\author{
di Maddalena Mazzocut-Mis \\ maddalena.mazzocut-mis@unimi.it
}

\begin{abstract}
Paul Valéry, in his "Discourse on Aesthetics", which he pronounced in 1936 in front of an audience of philosophers, reproached them, accusing aesthetics in particular of having pursued the abstract idea of beauty for too long, losing itself in the shadows of specialized terminology or in verbal games and thus forgetting the varied reality of works of art. Following an abstract idea of beauty means losing sight of the artistic object. It also means deluding oneself that the ugly is merely a counterbalance to a dominant category. But what does ugly mean? Its ambivalence lies precisely in a continuous oscillation between an evaluative and a descriptive use. There is a duplicity, so that on the one hand it expresses a negative judgment, and on the other hand - suspending its normal semantic destiny - it reclaims for itself some positivity. It is precisely from this positivity that the ugly begins its journey in the field of aesthetics.
\end{abstract}

Keywords: Aesthetics, Beauty, Ugly, Art

\section{Linee teoriche a partire dal Settecento ${ }^{1}$}

La riflessione sul brutto, come tema che concerne l'Estetica, ha origine nel Settecento europeo: Du Bos, Diderot, Burke, Lessing, tra gli altri, pur non dedicandovi un'opera specifica, si soffermano sul problema esaminandolo principalmente in un'ottica empirica e possiamo dire "psicologica".

\footnotetext{
${ }^{1}$ Questo articolo approfondisce alcune linee di ricerca già da me sondate in anni di studio e pubblicate in vari testi: cfr. Il gonzo sublime. Dal patetico al kitsch, Mimesis, Milano 2005; Estetica. Temi e problemi, Mondadori-Le Monnier, Firenze 2006 (la sezione dedicata a questo tema); Il senso del limite. Il dolore, l'eccesso, l'osceno, Mondadori-Le Monnier, Firenze 2009 e Itinerari estetici del brutto, Libreria Cortina, Milano 2011 (co-curatela con P. Giordanetti e G. Scaramuzza, autore di riferimento su questi temi). Si vedano i miei studi su G. Racioppi, Del Brutto nell'Arte, Unipress, Padova 1990 e su V. Hugo, Sul grottesco, Guerini, Milano 1990. Inoltre, cfr. i seguenti articoli, "Il piacere di piangere: il brutto, il sublime, l'orrore come godimento estetico", in M. Mazzocut-Mis (a cura di), Estetica della fruizione. Sentimento, giudizio di gusto e piacere estetico, Lupetti, Milano 2008, pp. 181-208; "Variazioni del brutto: dal patetico all'orrore", in M. Mazzocut-Mis (a cura di), Entrare nell'opera: $i$ Salons di Diderot, Mondadori-Le Monnier, Firenze 2012, pp. 16-40 e "Brutto", in G. Ferrario (a cura di), Estetica dell'arte contemporanea, Meltemi, Milano 2019, pp. 63-80.
} 
Jean-Baptiste Du Bos mette in luce la possibilità che il sentimento o, per usare un termine più moderno, l'emozione possa essere suscitata in modo più violento da ciò che nell'arte procura un dispiacere; Edmund Burke contemporaneamente a Moses Mendelssohn, ma su un versante teorico diverso - apre a una proficua teorizzazione sul sublime, sottolineando l'importanza dei sentimenti misti che amalgamo piacere e dispiacere; Gotthold Ephraim Lessing si interroga addirittura sui limiti della rappresentazione del brutto ${ }^{2}$.

In questi autori il brutto non assume un significato necessariamente antitetico al bello, ma si articola all'interno di una sfera più complessa e variegata legata anche alla mancanza di regolarità, proporzione, armonia, forma ed equilibrio. Queste caratteristiche possiamo dire "devianti" rispetto a una norma che rimane quella incarnata dal bello non inficiano la dignità del brutto ma anzi lo connotano.

L'estetica del Settecento si giostra inoltre all'interno di una tensione tra la tendenza all'unità e alla universalità e una forma di relativismo. Si tenta di trovare all'interno di una problematica estremamente controversa, che va dall'impressione soggettiva del piacevole, che esclude ogni regola, all'oggettivismo di un bello che si riscontra nella natura e negli oggetti, una definizione che si destreggi all'interno di altri due estremi: l'istinto - il bello è un "non so che" - e la ragione. Insomma, da un lato c'è un bello reale - se così non fosse, un discorso sul bello non sarebbe possibile - dall'altra c'è un bello relativo, che dipende dalla predisposizione del soggetto, dal contesto, dalla cultura, dagli usi e costumi. Se il bello reale si evince dall'intrinseca connessione, ordine e simmetria di ciascun oggetto (e qui il brutto verrebbe

\footnotetext{
${ }^{2}$ Cfr. J.-B. Du Bos, Riflessioni critiche sulla poesia e sulla pittura (1719), tr. it. a cura di M. Mazzocut-Mis e P. Vincenzi, Aesthetica, Palermo 2005; M. Mendelssohn, Sui sentimenti (1797), in Scritti di estetica, tr. it. a cura di L. Lattanzi, Aesthetica, Palermo 2004; E. Burke, Inchiesta sul bello e il sublime (1757), tr. it. a cura di G. Sertoli e G. Miglietta, Aesthetica, Palermo 2002; G. E. Lessing, Laocoonte (1766), tr. it. a cura di M. Cometa e G. Spatafora, Aesthetica Edizioni, Palermo 2007.
} 
escluso), quello relativo ha a che fare con un ordine di rapporti percepito dal soggetto (che invece ammette il brutto come valore).

Le qualità estetiche sono allora "relative" quando nascono dal rapporto tra soggetto e oggetto all'interno di un ambito definito. La bellezza non esiste al di fuori di una contestualizzazione o senza un soggetto che la esperisce. Siamo al cospetto di un'estetica esperienziale e in un certo senso sperimentale, nella quale appunto la tematica del brutto prende slancio.

Nella voce Brutto per l'Encyclopédie, Diderot riconosce l'impossibilità di giudicare il bello nel mondo della necessità. Il marmo - che nell'ambito della natura possiamo giudicare solo in base al principio di funzionalità - se è stato modellato dall'idea dell'artista, acquisisce un principio discriminante che corrisponde a quel modello ideale al quale l'artista si ispira e può essere giudicato bello o brutto indipendentemente dalla sua funzione. Ciò che è bello nell'arte, quindi, può risultare perfino brutto in natura. Una vecchia quercia secca e tutta storta, che il proprietario farebbe subito tagliare, è proprio quella che il pittore dipingerebbe, afferma Diderot ${ }^{3}$.

Il principio del bello e del bene e il loro contrario, il brutto e il male, non si trovano, così come li concepiamo, nell'ordine della natura, nella quale anche la mostruosità si giustifica. La natura crea individui capaci di esistere conformemente alle sue leggi, che l'uomo, anche in quanto artista, ha il dovere di comprendere e seguire da vicino. Un uomo brutto o meglio deforme - il brutto qui si confonde con l'imperfetto - sarà tale solo se comparato a un altro individuo che in modo più armonico risponde all'ordine della natura in generale; quello stesso uomo, se fosse l'unico essere vivente nell'universo, non potrebbe essere giudicato né bello né brutto, né perfetto né imperfetto 4 . «La

\footnotetext{
${ }^{3}$ Cfr. D. Diderot, Lettera sui sordomuti (1751), in D. Diderot, Lettera sui sordomuti e altri scritti, tr. it. a cura di E. Franzini, Guanda, Milano 1984, p. 48.

${ }^{4}$ Cfr. P. Sadrin, "L'article 'Laideur' de l"'Encyclopédie' ou les certitudes du désarroi", in S. Auroux, D. Bourel, C. Porset (éd. par), L’Encyclopédie, Diderot, l'esthétique, PUF, Paris 1991, pp. 261-266.
} 
natura non fa nulla di scorretto. Ogni forma, bella o brutta, ha la sua ragione d'essere, e in tutto ciò che esiste non c'è nulla che non sia come dev'essere» ${ }^{5}$.

Se la natura agisce solo sulla base di leggi organiche interne, il concetto di bello e di brutto presuppongono invece criteri stabiliti dall'uomo.

Bisogna subito mettere in chiaro che l'arte, pur partendo dalla natura, pur partendo da quell'albero secco che il pittore dipingerebbe mentre il proprietario taglierebbe, non è riproduzione servile della natura ma ricerca di un ideale pittorico, di un'unità artisticamente funzionale. Il modello incontra il particolare e il particolare assurge a modello. Questa la magia dell'arte per gli enciclopedisti.

Ora, non si può negare che, con poche eccezioni, fin dal Settecento ci sia stata una certa resistenza a definire il brutto e alla sua condivisione. Per quanto concerne il lato oggettuale, non si ha un termine univoco, ma una costellazione di riferimenti e rimandi all'informe, al deforme, alla vastità, all'estrema piccolezza, all'amorfo, a ciò che repelle e disgusta. Sul versante soggettivo, il riferimento a una emozione suscitata dal brutto, si confonde spesso con quella del sublime da un lato, escludendo qualsiasi anelito morale o legato all'elevazione dello spirito, o con quella del disgusto, ma senza arrivare agli estremi fisiologici.

Ora, l'emozione suscitata dal brutto ha comunque delle sue peculiarità: in primo luogo è difficile che non 'coinvolga', che non attiri un'attenzione partecipata, sebbene non tanto da far volgere il capo o da turare il naso, come nel caso del disgusto, o da far arretrare inorriditi, come nel caso del mostruoso o dell'orrore.

Un altro tema che è necessario considerare è lo slittamento - che non intacca però il giudizio di valore - dal rappresentare un oggetto brutto, seppur con grande maestria e consapevolezza tecnica, al rappresentare in modo

\footnotetext{
${ }^{5}$ D. Diderot, "Saggi sulla pittura", in D. Diderot I Salons, a cura di M. Mazzocut-Mis, seguiti dai Saggi sulla pittura e Pensieri sparsi, a cura di M. Modica, Bompiani, Milano 2021, p. 1489 .
} 
brutto, cioè con consapevole scarto di una possibilità esecutiva accurata e tecnicamente ineccepibile, un oggetto qualsiasi, anche bello. Uno slittamento essenziale che vede aprirsi uno squarcio sull'arte del Novecento. Insomma, possiamo avere un brutto della cosa e un brutto della sua resa nell'arte e questi due aspetti possono anche coincidere ma non necessariamente. "Brutto" può quindi essere il non riuscito, ciò che delude, ma anche il moralmente abietto oppure ciò che è tecnicamente imperfetto. Inoltre, ciò che comunemente è brutto in natura può acquisire una grande bellezza nell'arte, com'è noto almeno dalla Poetica di Aristotele in poi.

Tale complessità, che potrebbe sembrare un problema insormontabile, è invece un aspetto intrinseco alla nozione di brutto. C'è un brutto della cosa e un brutto della sua esecuzione, potremmo dire. Ed entrambi entrano nel novero delle possibilità artistiche.

Si arriva quindi al nodo centrale del problema. Attraverso la riflessione sulla rappresentazione del brutto, l'Estetica ha risposto a una domanda pressante: può esserci un godimento del brutto e, in caso affermativo, come si pone il nesso giudicativo?

Giudicare brutto un oggetto artistico, nel momento in cui brutto è categoria estetica, non ha necessariamente a che fare con un giudizio di valore ma con una modalità descrittiva che riesce a collocare l'oggetto in un ambito riconoscibile per il fruitore, il critico e il filosofo. A essere sinceri, però, il brutto sembra essere refrattario non solo a ogni piena positività, ma anche a ogni neutralità valutativa.

\section{Verso l'Ottocento}

Un testo che rappresenta un punto di svolta del brutto è Sullo studio della poesia greca ${ }^{6}$ di Friedrich Schlegel, che ha parlato di tale categoria come di

\footnotetext{
${ }^{6}$ Cfr. F. Schlegel, Sullo studio della poesia greca (1795), tr. it. a cura di G. Lacchin, Mimesis, Milano 2008. Il testo risale al 1795 e viene rielaborato nel 1823.
} 
un elemento specifico dell'arte moderna, indicando, quale segnale dell'incompletezza della filosofia dell'arte dei suoi tempi, il fatto che non ci fosse stato nessun tentativo di fondare una teoria del brutto, sebbene il bello e il brutto fossero ormai dei correlati inseparabili. Assenza di carattere, disordine, anarchia sono per Friedrich Schlegel gli elementi dominanti della poesia moderna, che non riesce più ad assolvere alle regole del bello, quali la compiutezza formale e l'armonia. Il caratteristico, l'individuale, l'interessante, il piccante, l'impressionante, l'insulso abbondano in un'arte che non è più 'bella'. In effetti il pubblico è ormai indifferente alla forma e assetato di contenuti. Chiede all'artista individualità interessante, che ha come contraltare la mancanza di universalità.

È però nell'ambito hegeliano che il brutto diventa uno stimolo essenziale del bello. Come ingrediente, esso coopera a determinare il comico, lo humour e il tragico. Potremmo quindi dire che, colorando il bello, lo rende interessante. Momenti nodali della tematica del brutto sono presenti nelle lezioni hegeliane sull'estetica ed è in questo periodo che al brutto viene dato quel nutrimento che lo trasforma in un problema teorico. Con il pensiero di Hegel si mette in evidenza ciò che ovviamente è sotto gli occhi di tutti: con il diffondersi del Cristianesimo e con l'allargarsi dei confini della rappresentabilità artistica, il brutto si diffonde pienamente nell'arte: i martiri e la stessa passione del Cristo ne sono un esempio più che evidente.

Ma c'è un altro processo che il brutto innesca arrivando fino ai nostri giorni, un processo più prosaico ma anche più duraturo: il brutto metterà in evidenza il dissonante, la mancanza di equilibrio, di ordine e di proporzione, l'uso della ripetizione e di converso della meraviglia, l'uso della banalizzazione e di converso della sorpresa, tutte modalità espressive che, quando incontreranno contenuti morali piccolo borghesi, daranno forma al melodrammatico fino al kitsch. Avvicinandosi al Novecento, il brutto assume anche il ruolo di rinnovamento e non di semplice valore negativo. Porta avanti il nuovo, un 
nuovo non complesso, che piace senza fatica perché diverte e mantiene alta l'attenzione.

Una nota a parte merita l'hegeliano Karl Rosenkranz che, nella sua Estetica del Brutto ${ }^{7}$ del 1853, mette in risalto la consapevolezza che bello e arte non coincidono necessariamente. Rosenkranz sostiene che esteticamente il brutto deve sempre riflettersi nel bello, nel quale trova la condizione della sua esistenza.

Scopo dell'arte è quindi la raffigurazione del bello, che è un 'assoluto', di contro al brutto che è solo un 'relativo'. Esiste un possibile riscatto del brutto, individuato nella caricatura, quale forma d'arte popolare, che può ristabilire l'armonia, funzionando da vertice dialettico in cui il brutto si rovescia nel comico. La caricatura, estrema disorganizzazione del bello, consiste nella manifestazione suprema del brutto, che quindi si scontra da una parte con il bello e dall'altra con il comico. Rosenkranz pone, quale vertice della produzione artistica, lo humour. Il comico ha la capacità di esprimere, meglio del tragico, lo sviluppo della libertà e, attraverso la serenità del riso, anche il male e il brutto. La caricatura è quindi un'esagerazione quantitativa e qualitativa, in grado di trasfigurare il negativo.

\section{Il Novecento e il brutto redentore}

Il brutto può anche contenere una speranza di redenzione. È Adorno a sostenere la necessità del salvataggio estetico di tale categoria. Nella sua postuma Teoria Estetica ${ }^{8}$, il significato estetico del brutto, che acquisisce una sempre più dichiarata autonomia, viene recuperato attraverso la sua carica dissacratoria. Se le ideologie dominanti proteggono un'arte pacificata e superficiale, non bisogna aver paura di guardare, sotto l'apparenza, una

\footnotetext{
${ }^{7}$ Cfr. K. Rosenkranz, Estetica del brutto (1853), tr. it. e introduzione a cura di E. Franzini, Aesthetica, Palermo 2004³.

8 T. L. W. Adorno, Teoria estetica (1970), tr. it. a cura di F. Desideri e G. Matteucci, Einaudi, Torino 2009.
} 
negatività che ha in sé una potenza pari a quella che si adopera per rimuoverla. Solo attraverso la liberazione dalla logica del dominio, che è un'oppressione sull'uomo, è possibile acquisire la capacità di produrre aspetti rappresentativi qualitativamente 'belli'. Il trionfo della ragione nasce quindi proprio negando la conciliazione e quindi negando il superamento del brutto attraverso una sublimazione nel bello. È un ribaltamento dei termini rosenkranziani, che prescrivono una conciliazione crescente, là dove il conflitto e la lotta si fanno più potenti.

Per Adorno il brutto risulta essere assai superiore al bello, che si riduce a mera convenzionalità senza conflitti. Una ragione non strumentalizzata può quindi portare alla luce le prerogative dell'esecrato, del deforme e del disarmonico. Adorno rivendica la positività del valore estetico del brutto e, facendone una sorta di 'redentore', porta al parossismo il ruolo rivoluzionario che dal romanticismo in poi aveva incarnato.

Possiamo quindi concludere che il brutto viene ad assumere un ruolo di risveglio se non di rinascita. Il ruolo del brutto è quello di indurci ad aprire gli occhi e risulta essere assai superiore al bello, che si riduce a mera convenzionalità senza conflitti. Il brutto mette in crisi e scardina l'edonismo estetico. Da qui la positività del valore estetico del brutto, della sua portata assolutamente rivoluzionaria.

Ma una domanda resta aperta: può esserci un godimento del brutto? La risposta è ovviamente sì. Il godimento del brutto è spesso più complesso e articolato di quello del bello. Un godimento che è stato descritto come un'emozione negativa, ma non tanto da farci ritrarre; un'emozione contraddittoria; un'emozione che può disturbarci, ma senza arrivare al disgusto. Ma tale godimento è ancora oggi così "interessante" o non risulta essere del tutto superato, tanto da essere solo una componente minoritaria di un'arte che non disdegna forme molto pronunciate di orrore e di disgusto?

In effetti, mischiato al tema della riproducibilità dell'opera d'arte e delle avanguardie, il brutto ha via via perduto la capacità di catalizzatore di istanze 
univoche. Dopo essere entrato nell'ambito dell'Estetica, ed essersi imposto in tutte le forme di rappresentazione, si è trasformato e moltiplicato, perdendo la sua specificità di padre generatore, confondendosi spesso con i suoi figli: il grottesco, il comico, il caricaturale, l'orrore, il disgustoso, il kitsch, ecc.

Non è dunque facile ai nostri giorni parlare di brutto. Sembra che questa categoria abbia fatto il suo tempo: dopo le avanguardie non è più portatore di nessuna rivendicazione e sempre più spesso assume accezioni semplicemente negative, là dove il sublime, l'orrore, il disgusto e il kitsch vivono invece ancora una fioritura accesa di colori. Il brutto è piuttosto il non definito, ciò che dà fastidio, il non bello (sempre che oggi si sappia che cosa sia il bello). L'aspetto di rottura e di contestazione che il brutto portava con sé risvegliava dei valori scomodi di cui era latore. Se ancora oggi evidenzia valori non pacificatori, tuttavia essi non sovvertono lo stato delle cose, non portano a cambiamenti radicali. Piuttosto stonano o lasciano solo un po' "stupiti” ma non certo attoniti.

Si faccia un esempio: se la Merda d'artista di Manzoni, nel 1961 voleva svelare i meccanismi e le contraddizioni del sistema dell'arte contemporanea, di fatto entrandone poi pienamente a far parte, se quindi quel brutto era nel suo stesso porsi provocatorio, la Turbo Cloaca di Wim Delvoye - un macchinario presentato nel 2000 al Museo di arte contemporanea di Anversa, che produce feci prevalentemente inodori - si pone immediatamente nel circuito artistico senza mai opporvisi, così come non si oppone al mercato e nemmeno all'uso di una tecnica raffinata il ciclo Shit del 2008 del fotografo Andres Serrano. In esso l'umore corporeo viene inquadrato lucidamente nella sua veridicità e contemporaneamente esasperato nella sua grandezza tanto da mascherarne la banalità. Lo stesso non si può dire dei 15 metri gonfiabili di Complex Shit di Paul McCarthy, sempre del 2008. Possiamo dunque chiederci se l'art rigolo o l'arte neo pop possano essere inserite nella categoria del brutto. Stando alle teorie di Rosenkranz, la risata "assolverebbe" il brutto elevandolo ad altro da sé, stornando l'effetto di rigetto. Oggi sappiamo bene 
che c'è risata e risata e che nella nostra contemporaneità il riso non "rettifica" ciò che nasce quale semplice irriverenza pantagruelica, come Complex Shit.

L'uso di umori corporali è pratica invalsa da molti anni. Se il brutto porta con sé almeno l'interessante', spesso negli esempi più contemporanei l'uso di escrementi o sperma o sangue mestruale non è altro che citazione.

\section{In conclusione}

È comunque difficile parlare di brutto senza dover anteporre delle considerazioni teoriche che lo posizionino tra le categorie estetiche.

Per la rivista «Domus», l'anno scorso, mi è stato chiesto di esprimermi riguardo a un caso che aveva fatto particolare scalpore: la presenza della modella Armine Harutyunyan - che non incarna gli stereotipi della bellezza contemporanea - sulle passerelle della moda.

Alcune considerazioni, tratte dall'intervista ${ }^{9}$, possono essere qui riprese a conclusione di questo articolo.

In primo luogo, constatavo che l'aggettivo brutto è, nel linguaggio comune, sempre e inequivocabilmente legato a un giudizio di valore negativo. In secondo luogo, rilevavo che l'autonomia del brutto, come valore estetico, non è una conquista assodata, anzi la strada è ancora molto lunga e complessa.

Sappiamo che l'arte ha sempre rappresentato il brutto e si è dilettata mettendo in scena l'orrore e il disgusto. Ma solo con il Settecento, l'Estetica si è interessata al brutto come categoria estetica con valore positivo. Il brutto diventa via via l'espressivo, contro un bello che nell'armonia e nell'equilibrio delle sue forme e dei suoi colori si fa superficiale conciliazione. Il brutto ci consente di guardare in faccia alle cose. Attribuire al brutto un valore positivo è stata la vera sfida della filosofia.

\footnotetext{
9 M. Mazzocut-Mis, intervista su «Domus»: L'importanza del brutto [on-line]. Pubblicato in data 9 settembre 2020, consultato in data 15 novembre 2021. Disponibile all'indirizzo: https://www.domusweb.it/it/arte/2020/09/09/limportanza-del-brutto.html.
} 
Ora, se l'arte ha acquisito la lezione - anzi l'ha insegnata all'Estetica nell'esperienza comune ancora si fatica ad attribuire al brutto un valore positivo. Nell'intervista avanzavo l'ipotesi che l'arte contemporanea fosse a tal punto pervasa dalla categoria dominante del kitsch - con la sua bellezza scontata e pacificata - e del trash - che al limite ingloba la portata dissacrante del brutto - da non riuscire a focalizzare il potenziale del brutto nella sua 'nudità'.

Eppure, se l'arte dà ormai per scontato il brutto, in un contesto diverso, come quello della moda, il brutto fa ancora parlare di sé. Il "non convenzionale" e il brutto - aggettivi attribuiti alla modella Armine Harutyunyan - ci danno da pensare, soprattutto quando rubano lo scettro a un bello stereotipato.

Se nell'arte il brutto è superato, nella moda ha ancora un valore dissacrante e pare svolgere il compito che Adorno gli aveva assegnato (o almeno ci prova). E non è un aspetto irrilevante se pensiamo che - a partire dall'Ottocento almeno - è la moda a dettare i canoni del bello e non certo l'arte.

Per trarre le somme, nei primi del Novecento la convinzione che il brutto si fosse ormai conquistato uno spazio a sé stante nel dominio dell'Estetica era data per acquisita e il brutto appariva nelle estetiche dell'empatia come il tocco di sensibilità e di espressività dato a un bello altrimenti asettico e ideale $^{10}$. Max Dessoir con un orientamento nettamente oggettivistico (opposto al soggettivismo delle estetiche dell'empatia) inquadrava il brutto come quella disarmonia dell'esistere di cui si alimenta anche il tragico.

$\mathrm{Su}$ un versante più fluido dal punto di vista teorico, Bataille, rovesciando Baudelaire, inneggiava al brutto capace di scardinare la noia attraverso il disgusto, poiché ogni bellezza è già guasta. «Com’è possibile - si chiedeva

\footnotetext{
${ }_{10}$ Nella sesta parte del primo volume della sua Estetica, Theodor Lipps dedica un capitolo al "brutto". Cfr. T. Lipps, Ästhetik. Psychologie des Schönen und der Kunst. I: Grundlegung der Ästhetik, Voss, Hamburg und Leipzig 1903.
} 
Bataille - che sotto la copertura della bellezza tanti artisti e scrittori non ci propongano altro che bruttezza? Come mai la ricerca del bello ha condotto al mostruoso e all'assurdo?» ${ }^{11}$.

Negli ultimi due decenni, si è fatta strada l'esigenza di mettere meglio a fuoco la natura delle emozioni negative provocate dalle opere d'arte legate al brutto e all'orrore e si tende a sottolineare la differenza nel modo in cui vengono vissute, rispetto a quelle suscitate da situazioni reali. È una via a mio parere molto proficua anche perché sembra convergere con i risultati e le vedute che emergono da indagini condotte anche al di fuori dell'ambito analitico e di carattere più speculativo ${ }^{12}$.

La perdita di attrattiva del brutto nel contesto specificamente artistico non deve quindi indurci ad abbandonare la sua indagine dal punto di vista teorico. Sappiamo bene che il fruitore non desidera il brutto, il mostruoso, l'orrore eppure li gode «violentemente e con dolore. Una passione». Il limite stesso del brutto, è «una frontiera» ${ }^{13}$, che viene continuamente valicata. Attraverso la riflessione sulla rappresentazione del brutto si può ancora tentare di rispondere a domande pressanti.

È in questo contesto che potrebbe essere proposta una ripresa della riflessione sul brutto. Certo, bisognerebbe saperne di più sulla pasta di cui sono fatte le emozioni negative di tipo finzionale. D'altro canto, psicologi, psichiatri e neurologi si aspettano da noi filosofi un indirizzo o una linea di ricerca che a mio parere potrebbe recuperare in modo proficuo la strada teorica percorsa dal brutto.

11 G. Bataille, L'arte, esercizio di crudeltà. Da Goya a Masson, tr. it. a cura di G. Zuccarino, Graphos, Genova 2000, p. 15 (in particolare cfr. G. Bataille, "La laideur belle ou la beauté laide dans l'art et la littérature", Critique, XXXIv, 1949).

12 Cfr. P. D'Angelo, La tirannia delle emozioni, il Mulino, Bologna 2020 e M. Mazzocut-Mis, "The Pleasure of Weeping: The Novelty of a Research", in P. Giacomoni, N. Valentini, S. Dellantonio (ed. by), The Dark Side: Philosophical Reflections on the "Negative Emotions", Springer Nature Switzerland, Cham 2021, pp. 159-175.

13 J. Kristeva, Poteri dell'orrore. Saggio sull'abiezione (1980), tr. it. di A. Scalco, Spirali, Milano 1981, p. 11. 


\section{Nota bibliografica}

ADORNO, Theodor Ludwig Wiesengrund, Teoria estetica (1970), tr. it. a cura di F. Desideri e G. Matteucci, Einaudi, Torino 2009.

BATAILLE, Georges, "La laideur belle ou la beauté laide dans l'art et la littérature", Critique, XXXIV, 1949.

—, L’arte, esercizio di crudeltà. Da Goya a Masson, tr. it. a cura di di G. Zuccarino, Graphos, Genova 2000.

BURKE, Edmund, Inchiesta sul bello e il sublime (1757), tr. it. a cura di G. Sertoli e G. Miglietta, Aesthetica, Palermo 2002.

D’ANGELO, Paolo, La tirannia delle emozioni, il Mulino, Bologna 2020

DIDEROD, Denis, "Saggi sulla pittura", in D. Diderot, I Salons, a cura di M. Mazzocut-Mis, seguiti dai Saggi sulla pittura e Pensieri sparsi, a cura di M. Modica, Bompiani, Milano 2021.

—, "Lettera sui sordomuti" (1751), in D. Diderot, Lettera sui sordomuti e altri scritti, tr. it. a cura di E. Franzini, Guanda, Milano 1984.

DU BOS, Jean-Baptiste, Riflessioni critiche sulla poesia e sulla pittura (1719), tr. it. a cura di M. Mazzocut-Mis e P. Vincenzi, Aesthetica, Palermo 2005.

GIORDANETTI, Piero, MAZZOCUT-MIS, Maddalena, SCARAMUZZA, Gabriele (a cura di), Itinerari estetici del brutto, Libreria Cortina, Milano 2011.

HUGO, Victor, Sul grottesco (1827), tr. it. di M. Mazzocut-Mis, Guerini, Milano 1990.

KRISTEVA, Julia, Poteri dell'orrore. Saggio sull'abiezione (1980), tr. it. di A. Scalco, Spirali, Milano 1981. 
LESSING, Gottlieb Ephraim, Laocoonte (1766), tr. it. a cura di M. Cometa e G. Spatafora, Aesthetica Edizioni, Palermo 2007.

LIPPS, Theodor, Ästhetik. Psychologie des Schönen und der Kunst. I: Grundlegung der Ästhetik, Voss, Hamburg und Leipzig 1903.

MAZZOCUT-MIS, Maddalena, Il gonzo sublime. Dal patetico al kitsch, Mimesis, Milano 2005.

—, Estetica. Temi e problemi, Mondadori-Le Monnier, Firenze 2006.

—, "Il piacere di piangere: il brutto, il sublime, l'orrore come godimento estetico", in M. Mazzocut-Mis (a cura di), Estetica della fruizione. Sentimento, giudizio di gusto e piacere estetico, Lupetti, Milano 2008, pp. 181-208.

—, Il senso del limite. Il dolore, l'eccesso, l'osceno, Mondadori-Le Monnier, Firenze 2009.

—, "Variazioni del brutto: dal patetico all'orrore", in M. Mazzocut-Mis (a cura di), Entrare nell'opera: $i$ Salons di Diderot, Mondadori-Le Monnier, Firenze 2012, pp. 16-40.

-, "Brutto", in G. Ferrario (a cura di), Estetica dell'arte contemporanea, Meltemi, Milano 2019, pp. 63-80.

—, Maddalena, intervista su "Domus": L’importanza del brutto [on-line]. Pubblicato in data 9 settembre 2020, consultato in data 15 novembre 2021. Disponibile all'indirizzo: https:/www.domusweb.it/it/arte/2020/09/09/limp ortanza-del-brutto.html.

—, "The Pleasure of Weeping: The Novelty of a Research", in P. Giacomoni, N. Valentini, S. Dellantonio (ed. by), The Dark Side: Philosophical 
Reflections on the "Negative Emotions", Springer Nature Switzerland, Cham 2021, pp. 159-175.

MENDELSSOHN, Moses, "Sui sentimenti" (1797), in Scritti di estetica, tr. it. a cura di L. Lattanzi, Aesthetica, Palermo 2004.

RACIOPPI, Giacomo, Del Brutto nell'Arte, Unipress, Padova 1990.

ROSENKRANZ, Karl, Estetica del brutto (1853), tr. it. e introduzione a cura di E. Franzini, Aesthetica, Palermo 20043.

SADRIN, Paul, "L'article 'Laideur' de l"Encyclopédie' ou les certitudes du désarroi”, in S. Auroux, D. Bourel, C. Porset (éd. par), L’Encyclopédie, Diderot, l'esthétique, PUF, Paris 1991, pp. 261-266.

SCHLEGEL, Friedrich, Sullo studio della poesia greca (1795), tr. it. a cura di G. Lacchin, Mimesis, Milano 2008.

\section{Nota biografica}

Maddalena Mazzocut-Mis è Professore Ordinario di Estetica, saggista e drammaturga. Insegna Estetica ed Estetica della musica e dello spettacolo presso il Dipartimento di Beni culturali e ambientali dell'Università degli Studi di Milano. È stata Visiting Professor e Academic Visitor nelle Università di Aix-Marseille e Avignonet des Pays de Vaucluse (Francia), di York, Oxford (UK), di Galati (Romania), di Malaga (España). È vincitrice di numerosissimi finanziamenti per la ricerca scientifica in Italia e in Europa. Tra le sue ultime monografie nel campo della saggistica, si ricordano: Le Monstre. L'anomalie et le difforme dans la nature et dans l'art (Peter Lang, Bern 2018); Philosophy of Picture. Denis Diderot's Salons (Peter Lang, Bern 2018); Frammenti di sipario (Mimesis, Milano 2019) e Teatro da leggere. Mito e conflitto (Le Monnier, Firenze 2021). 